\title{
Assessment of fetoplacental function by biochemical determinations
}

\author{
T. CHARD
}

From St Bartholomew's Hospital Medical College and the London Hospital Medical College, London

For 50 years the emphasis of obstetric management has shifted from the mother to the unborn child. Maternal mortality has fallen from rates of 4 per 1000 or greater to rates of $0 \cdot 2$ per 1000 , so that the chances of a woman dying during pregnancy are hardly more than those if she were not pregnant over the same time period (Registrar General, 1972). But perinatal mortality at around 25 per 1000 continues to be well above the possible minimum; furthermore there will be two or three children severely damaged for every one which dies (Chard, 1974a). The prevention of perinatal mortality and morbidity might, in part, be achieved by intensification of the existing clinical approach. Yet the scope of purely clinical methods has probably reached its peak, and most advances are certain to involve laboratory-orientated technology, including electronic and biochemical methods for the assessment of fetal wellbeing and growth.

With the exception of congenital abnormality, the placenta is the common denominator in many of the conditions which threaten fetal wellbeing. For the whole period of intrauterine life, the placenta has to act as lung, gut, kidney, and as a barrier to infection. Most of the biochemical methods are directed towards the study of this organ and its relationship with the fetus.

\section{Fetoplacental Synthetic Functions}

The main function of the placenta is the transfer of nutrients and waste products between the mother and fetus. A test that measured this function would be of immense clinical value; attempts to develop the appropriate technology have so far not been generally applied (Garrow and Douglas, 1968; Chatfield et al, 1975). As an alternative, therefore, most current methods are tests of placental synthesis.

The placenta produces an extensive range of substances, including enzymes and hormones, which differ either qualitatively or quantitatively from those in the non-pregnant adult (table I). The steroid products of the placenta are identical in structure to

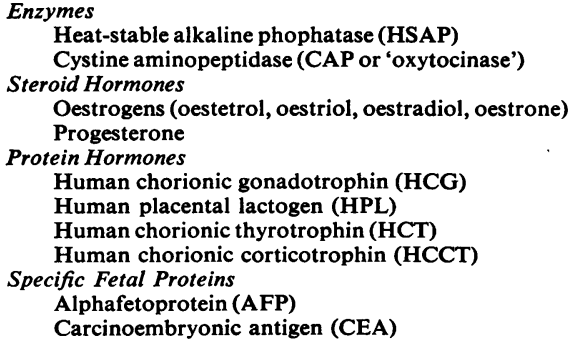

Table I Products of the human fetoplacental unit

those found in the non-pregnant state. The proteins, by contrast, are analogous to but not necessarily identical with normal adult products: thus, placental alkaline phosphatase remains active after heating at 56 to $60^{\circ} \mathrm{C}$; placental lactogen is similar chemically to both prolactin and pituitary growth hormone, but has only minimal biological activity when compared with the last two.

Though all the placental products listed in table I are synthesized and released by the syncytial layer of the trophoblast, some, such as oestriol, depend on a supply of fetal precursors (fig 1). Certain parts of the trophoblast may be more active than others and there is evidence for specialization into thin areas adjacent to fetal capillaries, which are responsible for transport, and thicker areas, not adjacent to capillaries, which are responsible for synthesis (Burgos and Rodriguez, 1966). The steroids can move relatively freely between mother and fetus, though they are extensively metabolised in the trophoblast; by contrast protein hormones are secreted mainly into the maternal circulation and the concentrations of HPL and HCG in the fetus are 100 times less than those in maternal peripheral blood. These observations indicate that the basement membrane or the fetal capillary endothelium forms a barrier to the passage of the larger molecules. Specific placental products also occur in amniotic fluid; the route of entry is not certain, and is likely to vary for different materials. 


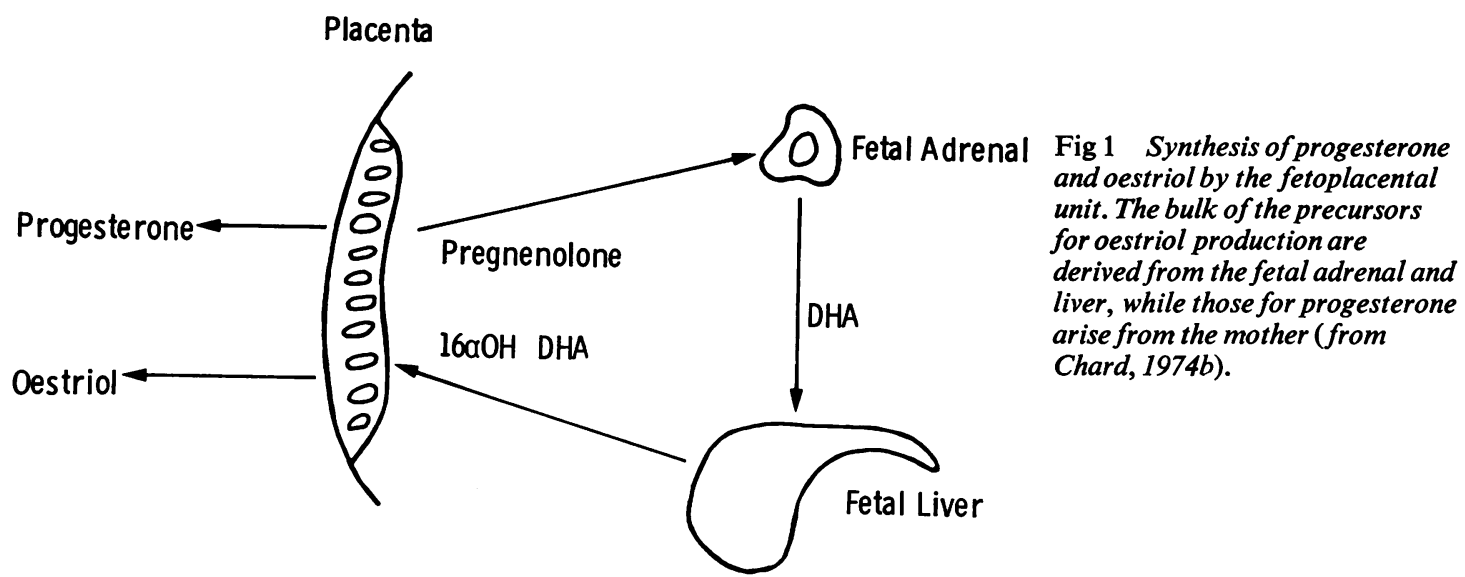

The concentration of fetoplacental products in the mother will vary with the amount produced, and also with maternal metabolism and excretion. It might be anticipated that the product of a given fetoplacental unit would give half the circulating level in a 12-stone woman as it would be in a 6-stone woman. Body weight is rarely considered in the interpretation of hormonal tests of placental function, though it might be of considerable practical significance. Furthermore, it is often not realized that the excretion of oestrogen conjugates in urine is dependent on urine flow rate: the total excretion in 24 hours is greater if the patient excretes 2 litres rather than 1 litre of urine. In addition, the rate of excretion in urine can vary with renal function: low urine oestrogens in a patient with preeclampsia may reflect the associated renal lesion as well as fetoplacental dysfunction.

The control mechanisms for the production of specific placental products are virtually unknown. In adult life the release of hormones is characterized by well defined feedback systems on which are based several dynamic tests of function. In the placenta such factors are much less obvious. Thus, though HPL probably plays a role in carbohydrate and lipid metabolism, there is only a minimal response to intravenous glucose (Pavlou et al, 1973). Trophoblastic synthesis of hormones seems to be virtually autonomous, in a manner similar to that of some adult tumours, though it can be changed by an alteration in the supply of precursors; for instance, oestriol synthesis is reduced in association with corticosteroid therapy (Oakey, 1970) due to suppression of the fetal pituitaryadrenal axis and a decrease in the synthesis of DHA (fig 1). Production of placental hormones may also be influenced by the rate of blood flow in the intervillous space (McNeilly et al, 1976); this is particularly important in considering the mechanism of reduced levels in pathological pregnancies.

The functional significance of most placental hormones is doubtful. On many occasions it has been suggested that the placental steroids play an important part in the maintenance of pregnancy and the onset of spontaneous labour (see Klopper and Gardner, 1974), but the nature of the precise action is as yet uncertain. It has also been suggested that the specific hormones of the placenta are responsible for the metabolic alterations observed in the mother during pregnancy. It is difficult to attribute a specific change to a specific hormone: thus changes in carbohydrate and lipid metabolism may be due to many factors, both steroid and protein, and no one hormone can be pointed out as preeminent.

\section{The Clinical Evaluation of Biochemical Tests of Fetoplacental Function}

Much effort is spent on the identification of new parameters of fetoplacental function when, in fact, the main requirement is for detailed evaluation and refinement of tests which are already available. Probably most of the substances shown in table I would be an effective index of fetoplacental wellbeing, but the convenience and precision of measurement is probably more important than the nature of the material.

The practical use of tests of fetoplacental function has been retarded rather than advanced by much of the published information. The literature consists, with some notable exceptions, of case reports which are of little value to the obstetrician because they do not reflect the bulk of day-to-day clinical practice. Cases are often presented in which striking clinical events are associated with equally dramatic bio- 
chemical changes, yet often laboratory investigations have played little part in the practical management of these patients. Three criteria should be fulfilled if fetoplacental hormone determinations are to be useful in clinical practice: the definition of a normal range, the definition of the range for abnormalities and its relation to fetal outcome, and the interpretation of results from an individual subject related to the range of normal and abnormal.

NORMAL RANGE

A normal range for a fetoplacental product should be based on sufficient numbers of subjects and samples (at least 100 for each week of pregnancy), and the criteria for a normal pregnancy should be clearly defined. The optimal presentation of the range is as a mean, plus and minus two standard deviations; this is easily understood by the clinician and is comparable from one group of workers to the next. It is important to realize that, with most tests, the range is not normally distributed, but, instead, is skewed at the upper end by the occurrence of a few relatively high values (fig 2 ). Thus, the mean and the range should be calculated either as centiles or after logarithmic or square root transformation of the data. The resultant range shows less variance below the mean than above it, and it is in the former that most values of clinical interest will lie.

The variance of the normal range reflects on the potential clinical use of a test. Where normal variation is considerable, substantial overlap with the abnormal is likely and the test is unlikely to be of value. The variation observed has two parts: one due to the biological differences between individuals, and the other to errors in the method of estimation (fig 3). Variations due to biological spread can only be reduced by subdividing the population into clinically recognizable groups, but assay variation can be improved by detailed attention to laboratory procedure and sample collection. A unit setting up a test for the first time should always establish its own normal range, since even with well established methodology small variations in the population examined can yield ranges which differ strikingly from those presented by other groups.

THE RANGE IN ABNORMALITIES

The aim of a laboratory test is to give information on the wellbeing of a pregnancy which cannot be obtained by clinical means. Tests relating to the maternal disease are helpful only if they provide evidence that enables the clinician to assess fetal prognosis. It is important to consider both the normal range and the range for the abnormality. A good example is provided by the study of HPL levels in diabetic pregnancy (Ursell et al, 1973). Cases with

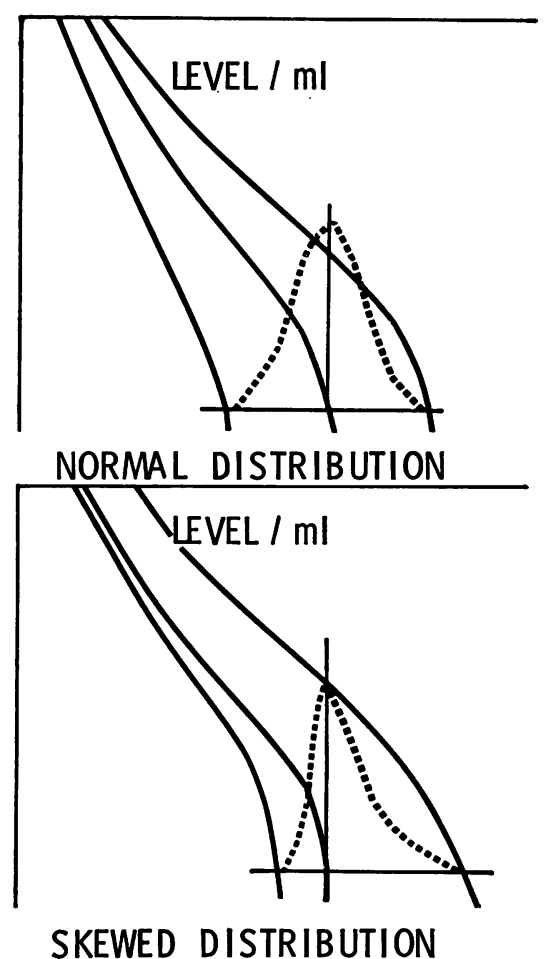

Fig 2 Diagrams showing the normal range for a test of placental function (concentrations of substance on horizontal axes; stage of gestation on vertical axes). $A$ 'normal' distribution with equal variations on both sides of the mean is shown in the upper diagram. A 'skewed' distribution with greater variation above the mean than below it is shown in the lower diagram. The latter type of distribution always occurs with tests of placental function (from Chard, 1974b).

a satisfactory fetal outcome have levels elevated above the normal (fig 4); cases with an unsatisfactory outcome have levels lower than those of 'normal' diabetics, but much less so when compared with the normal range (fig 5). If the normal range were the only criterion, most of the clinical use of this test in diabetes could not be realized.

\section{Results in individuals}

The statistical analysis of a fetoplacental function test is usually carried out retrospectively on a complete population, and the application of the test to the prospective analysis of results in individual patients is largely unexplored. A common approach is subjective assessment of the biochemical results by the obstetrician; this is acceptable when the results are very striking, but less so when the 


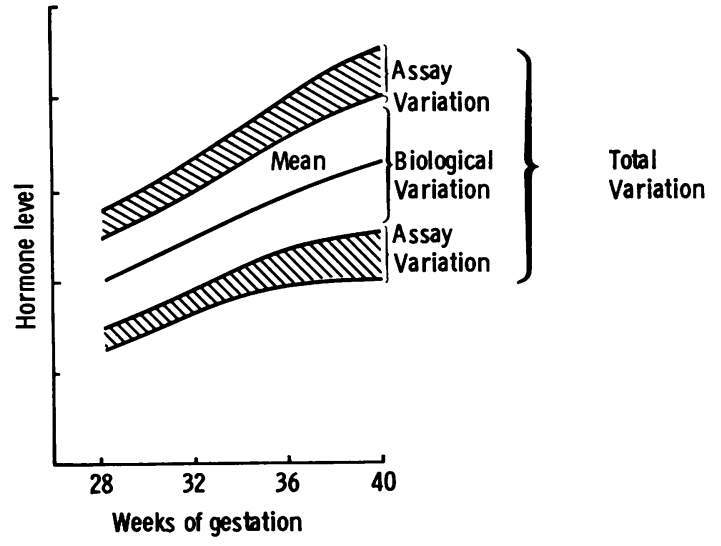

Fig 3 Diagram showing the two sources of variation in the normal range for a placental function test: biological and assay variation are additive, and every effort should be made to reduce assay variation to a minimum (from Chard, 1974b).

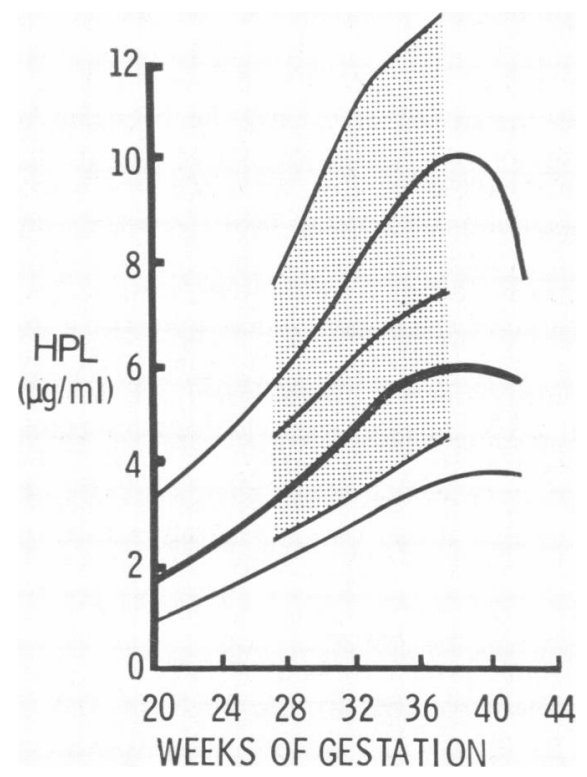

Fig 4 The range of maternal plasma HPL values in diabetic women with uncomplicated pregnancies (mean \pm 2 sd), superimposed on the normal range (from Ursell et al, 1973).

abnormality is borderline. Undoubtedly, the most efficient method of assessment is to express the results in terms of 'risk': arbitrary limits are set on the basis of continuous data collection and a risk factor is assigned to cases where values fall between

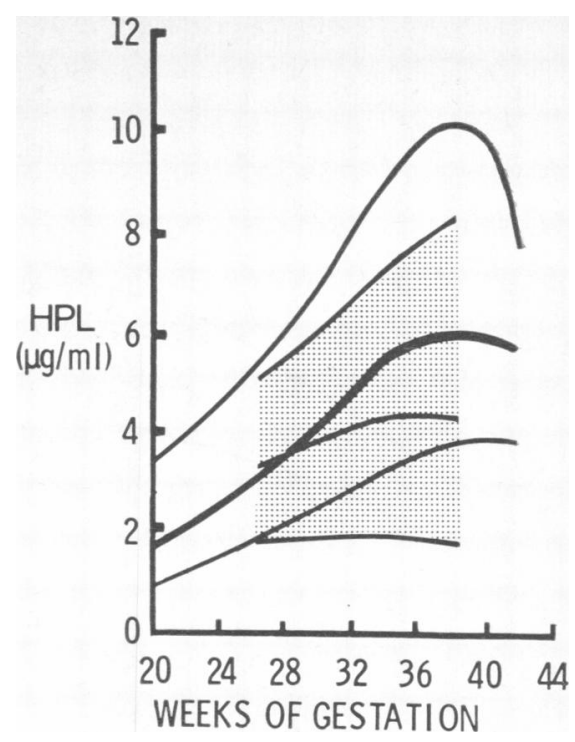

Fig 5 The range of maternal plasma HPL values in diabetic women with complicated pregnancies (mean \pm 2 $s d$ ), superimposed on the normal range (from Ursell et al, 1973).

or below these limits. Using this approach it is often found that the accumulation of small risk factors, eg, levels of placental hormones at the lower end of the normal range (fig 6), may together create a situation of high risk which demands immediate attention from the obstetrician. It must be appreciated that a biochemical measurement is no more definite than, for instance, measurement of blood pressure; this has, in the past, produced disillusion with techniques which were over-enthusiastically introduced.

The charting of serial levels in individual patients is important since high risk may be indicated by a progressive fall. However, this is a rare event in practice and when it is observed, it is important to exclude the possibility that it reflects random variations of the method or the patient. A considerably more important function of serial levels is the added confidence that they give to the relationship between individual results and those of the whole population. A series of values at the lower limit of normal may suggest a greater risk than one isolated value which is far lower.

\section{Specific Biochemical Tests of Fetoplacental Function}

All of the materials shown in table $I$ appear in maternal blood and/or urine and in theory measurements of any might be useful as tests of fetoplacental 


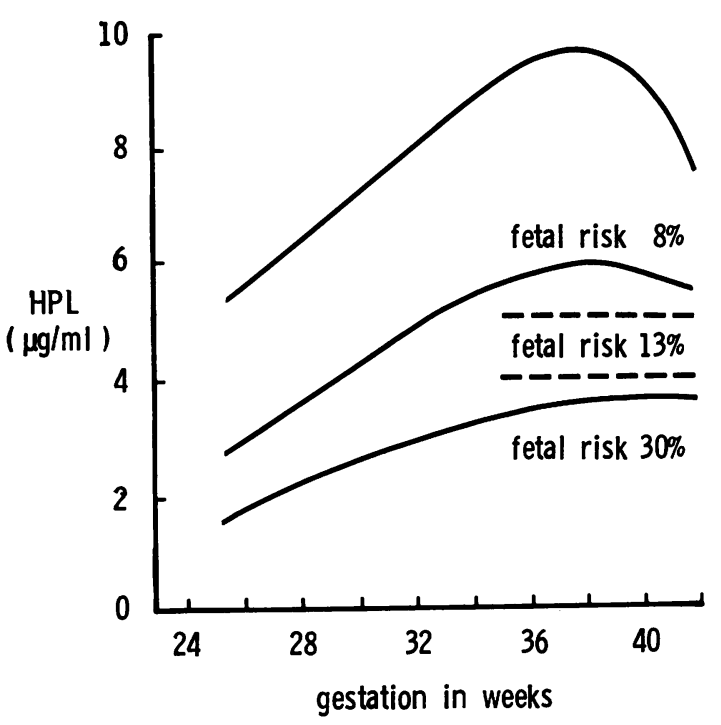

Fig 6 The risk of fetal distress and/or neonatal asphyxia in pregnancies which are normal by clinical examination, but maternal HPL levels are within the limits shown by the interrupted lines. Note that fetal risk increases even with levels in the low normal range (redrawn from Letchworth and Chard, 1972).

wellbeing. In practice a number of secondary criteria determine their clinical value (table II).

\section{PLACENTAL ENZYMES}

Cystine aminopeptidase (CAP or 'oxytocinase') and heat-stable alkaline phosphatase (HSAP) are specific placental enzymes which should satisfy most of the criteria of table II. The estimation of CAP can be made so rapid and simple that on technical grounds it would be the test of choice in most laboratories (Tovey et al, 1973). Enzyme determinations are not widely applied. In a very full review of the literature Shane and Suzuki (1974) concluded that measurement of HSAP was of no proven clinical value. The evidence with respect to CAP is almost purely anecdotal and no conclusion is possible. The difficulty probably arises because CAP and HSAP are determined in terms of substrate activity rather than by mass; such activity can measure a heterogeneous

1 The method of measurement should be rapid, simple, and precise.

2 The half-life in the mother should be short so that any change in production is rapidly reflected in the concentration in blood or urine.

3 The circadian and day-to-day variation should be minimal.

4 The test should provide a distinction between normal and abnormal cases.

Table II Criteria for the clinical use of biochemical tests of fetoplacental function range of molecules, and apparently minor variations in technique, such as the temperature of the reaction, can lead to substantial variation in the results. The variation will produce differences between laboratories, and differences between separate assays in the same laboratory or even between consecutive samples in the same assay. If the methodology could be improved it is likely that measurement of placental enzymes could provide useful information of fetal wellbeing.

\section{SPECIFIC FETAL PROTEINS}

Alphafetoprotein

Alphafetoprotein (AFP) is the major circulating protein of the early human fetus, reaching peak levels at the 12th to 14th week of intrauterine life (Gitlin and Boesman, 1966). It appears in maternal blood, the levels rising progressively to reach a peak at the 32nd to 34th week of gestation and then declining until term (Leek et al, 1975). The source of maternal AFP is uncertain though it is probably derived from the fetus by transfer across the placenta and membranes. Measurement in biological fluids is carried out by immunological methods, the most widely applied of which is radioimmunoassay.

Measurement of AFP may be of value in the diagnosis of fetal complications in late pregnancy. The maternal blood values are often grossly elevated in association with fetal death at any stage of pregnancy, and the increase may occur even before fetal death (Seppälä and Ruoslahti, 1973); the increased concentrations under these circumstances are probably due to fetomaternal haemorrhage. Sometimes, however, high levels are found in a normal pregnancy, so there is need for caution in the interpretation of results. The place of this estimation is probably in high risk pregnancies, provided that it can be conclusively shown that its use contributes significantly to fetal salvage.

\section{Carcinoembryonic antigen}

Carcinoembryonic antigen (CEA) is a specific glycoprotein secreted by the fetal gut and, in the adult, by tumours of the gut and of certain other sites (Gold and Freedman, 1965). High concentrations are found in the fetus and small amounts in maternal blood. Measurement is by radioimmunoassay.

The only clinical application has been the estimation of CEA in amniotic fluid as a guide to fetal distress (Goldenberg et al, 1972). The concept is that measurement of levels of CEA should indicate the presence of meconium in amniotic fluid, present in quantities too low to be seen by the naked eye. Attractive though this is as a concept, its value in practice still remains unproven. 


\section{FETOPLACENTAL STEROID HORMONES}

\section{Progesterone}

Some $15 \%$ of the progesterone synthesized by the placenta is excreted in maternal urine as pregnanediol. Determination of this has been used as a test of placental function, but the measurement is timeconsuming and this, together with the problems of sample collection, explains the limited published evidence on this subject.

Chemical determination of pregnanediol in urine is likely to be replaced by direct radioimmunoassay of progesterone in blood. There is as yet no substantial literature on the clinical application of this technique, though the significance of blood progesterone values is likely to be similar to that of placental lactogen (see below).

\section{Oestrogens}

Oestriol is quantitatively the most important of the major oestrogens in late pregnancy. It is synthesized by the placenta from fetal precursors (fig 1) and thus reflects the function of both fetus and placenta together rather than, as with other materials, the function of the placenta alone (Diczfalusy and Mancuso, 1969; Beling, 1971).

Most work on oestrogens has, until recently, been based on determination in a 24-hour specimen of urine. This has the great disadvantage that the collection is often inaccurate. Measurement of oestriol in maternal blood is increasingly widely used and may well replace the use of urinary oestriol in obstetric practice. This has been made possible by the introduction of radioimmunoassay which is sufficiently sensitive to permit precise estimation of the relatively low circulating levels.

Most of the published evidence on the clinical use of oestrogen measurement concerns the urinary oestrogens. A typical range of normal values is shown in figure 7; this range will vary in different laboratories, and every group should establish their own normal values. Low or subnormal oestrogen values reflect an increased risk to the fetus in several of the common complications of pregnancy, including intrauterine growth retardation (Yousem et al, 1966; Heys et al, 1968), preeclampsia (Macleod et al, 1967; Bjøro, 1972), and diabetes mellitus (Evans et al, 1972). In prolonged pregnancy, subnormal or decreasing levels are an indication for immediate delivery (Smith et al, 1966; Lundvall and Stakemann, 1966; Beischer et al, 1969). Urinary oestriol levels are low in most cases of fetal death (Frandsen and Stakemann, 1960; Heys et al, 1968). However, the test is not always useful because, with acute conditions such as abruptio placentae, the levels are often normal until death has already occurred. An important potential use of oestrogen

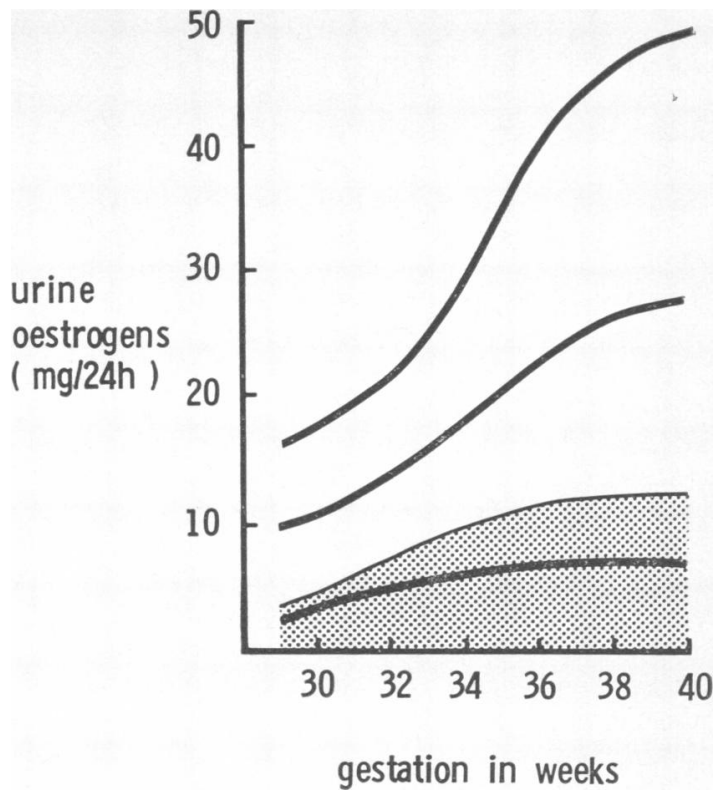

Fig 7 A typical normal range for urinary oestrogens in late pregnancy. The shaded area indicates the levels which suggest fetal risk.

measurement is in the prediction of fetal risk in an apparently normal pregnancy (Beischer et al, 1968). The use of biochemical procedures as screening tests is further discussed below.

Information on the clinical use of blood oestrogen determination is still relatively sparse. In addition, it is still not certain which oestrogen fraction should be measured: the principal candidates are unconjugated oestriol and total oestriol (conjugated and unconjugated) (see Klopper and Shaaban, 1973). Clinical interpretation will probably be similar to that of urine oestrogens, though the measurement in blood is hoped to prove more efficient as a parameter of fetal welfare because of better reproducibility of sample collection and estimation. Specific oestradiol determinations have interesting possible applications. A considerable rise in oestradiol levels has been shown before the onset of spontaneous premature labour (Tamby Raja et al, 1974); if this is confirmed, the measurement could be of great value in the prediction and prevention of a complication which is still the most important cause of perinatal mortality and morbidity.

PLACENTAL PROTEIN HORMONES

Human chorionic gonadotrophin

Human chorionic gonadotrophin (HCG) is a specific placental glycoprotein which is biologically 
and chemically similar to pituitary luteinizing hormone. Levels in maternal blood and urine reach a peak between the seventh and eighth weeks of pregnancy. Although measurement of HCG by radioimmunoassay or simple immunological agglutination systems is extensively used for the diagnosis of early pregnancy, and for the diagnosis and management of trophoblastic tumours, there is little information on the use of HCG estimations as a test of fetal welfare (Brody, 1969).

\section{Human placental lactogen}

Human placental lactogen (HPL) is a specific placental protein which is immunologically and chemically similar to pituitary growth hormone and prolactin. Levels in maternal blood rise progressively during pregnancy reaching a plateau after the 35th week (fig 8). The measurement of HPL by radioimmunoassay is simple, rapid and precise, and is increasingly widely used in the diagnosis of complications in both late and early pregnancy (see Chard, 1975).

The normal range of HPL levels in maternal blood (fig 8) is less wide than that of either urine or plasma oestrogens. Low normal or subnormal concentrations reflect increased fetal risk in complications such as threatened abortion (Niven et al, 1973),

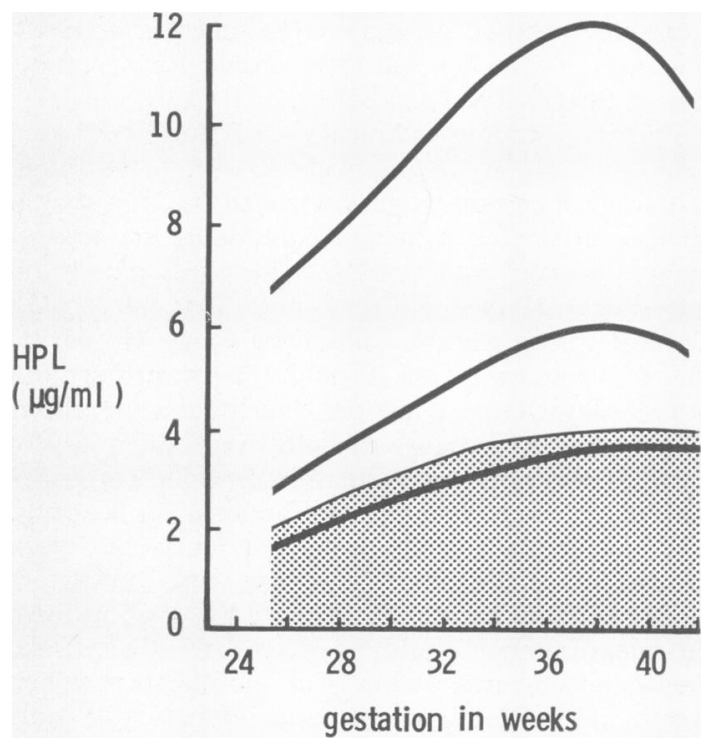

Fig 8 A typical normal range for maternal plasma $H P L$ in late pregnancy. The shaded area indicates the levels which suggest fetal risk. Note that the normal range is narrower than that for urinary oestrogens, and that the area in which fetal risk can be diagnosed is relatively greater. preeclampsia (Spellacy et al, 1971; Keller et al, 1971; Lindberg and Nilsson, 1973), and diabetes mellitus (Ursell et al, 1973) (see also figs 4 and 5). HPL levels are related to fetal weight, but they are less efficient in the identification of intrauterine growth retardation than the fetus-dependent oestrogens. The levels are often low before fetal death (Spellacy et al, 1971; Ward et al, 1973). In rhesus isoimmunization, high levels of HPL before the 26th week of pregnancy suggest that the condition is likely to be severe (Ward et al, 1974). Low levels of HPL may predict fetal complications in the apparent absence of clinical abnormality (Letchworth and Chard, 1972; England et al, 1974); values of less than $4 \mu \mathrm{g} / \mathrm{ml}$ after the 35th week are associated with a high incidence of fetal distress during labour and neonatal asphyxia after delivery.

Other placental protein hormones (table I) have not been examined as tests of fetal welfare. It seems likely that their significance would be similar to that of HPL.

DYNAMIC TESTS OF PLACENTAL HORMONE SYNTHESIS

The administration of dehydroepiandrosterone sulphate (DHA) to the mother leads to an increase in placental oestrogen synthesis (Lauritzen, 1967) (see fig 3). This procedure might provide a test of placental reserve capacity but considerably more work is needed before it can be used in practice.

\section{Conclusions}

Biochemical tests of fetoplacental function are an integral part of the care of the high-risk pregnancy. In addition, tests of this type are promising as screening procedures for all pregnancies; clinical examination will detect only a proportion of at-risk pregnancies, and it is likely that a biochemical test (oestrogens or HPL) will identify some of the remaining cases. It is possible that tests of this type may become as routine a part of the antenatal visit as is the testing of urine or the measurement of blood pressure.

Of existing tests, measurement of oestrogens (oestriol) in maternal urine and HPL in maternal blood are of most practical use because the techniques are well established and there is a full range of published evidence on which to base their interpretation. Blood is likely to replace urine as the material for oestrogen determination when further work has been carried out.

Several future advances may be anticipated. Biochemical tests other than those measuring oestrogens and HPL will be examined, though it is perhaps unlikely that these will have any significant advantages over established tests. More important, 
clinicians will become increasingly aware of the uses and the limitations of biochemical tests of fetal welfare, for, as with clinical observations, scientific techniques yield results which are relative rather than absolute. Finally, it will be appreciated that in some cases the biochemical result is the sole indicator of fetal risk, and that it is then operating as a primary diagnostic tool.

\section{References}

Baillie, P. (1974). Non-hormonal methods of antenatal monitoring. Clinics Obstet. Gynec., 1, 105.

Beischer, N. A., Bhargava, V. L., Brown, J. B., and Smith, M. A. (1968). The incidence and significance of low oestriol excretion in an obstetric population. J. Obstet. Gynaec. Brit. Cwlth, 75, 1024-1033.

Beischer, N. A., Brown, J. B., Smith, M. A., and Townsend, L. (1969). Studies in prolonged pregnancy. II. Clinical results and urinary estriol in prolonged pregnancy. Amer. J. Obstet. Gynec., 103, 483-495.

Beling, C. (1971). Estrogens. In Endocrinology of Pregnancy, (edited by F. Fuchs and A. Klopper, pp. 32-65. Harper and Row, New York.

Bjøro, K. (1972). Oestriol assay in obstetric practice. Acta endocr. (Kbh.), Suppl. 161.

Brody, S. (1969). Protein hormones and hormonal peptides from the placenta. In Foetus and Placenta, edited by $\mathrm{A}$. Klopper and E. Diczfalusy, pp. 299-411. Blackwell, Oxford.

Burgos, M. H., and Rodriguez, E. M. (1966). Specialized sones in the trophoblast of the human term placenta. Amer. J. Obstet. Gynec., 96, 342-356.

Chard, T. (1974a). The fetus at risk. Lancet, 2, 880-883.

Chard, T. (1974b). The hormonal assessment of fetal and placental function. Clinics Obstet. Gynec., 1, 85-102.

Chard, T. (1975). Human Placental Lactogen Levels as a Guide to Fetal Well-being. (Medical Monographs, 8). Radiochemical Centre, Amersham.

Chatfield, W. R., Rogers, T. G. H., Brownlee, B. E. W., and Rippon, P. E. (1975). Placental scanning with computerlinked gamma counter camera to detect impaired placental blood flow and intrauterine growth retardation. Brit. med. J., 2, 120-122.

Diczfalusy, E. and Mancuso, S. (1969). Oestrogen metabolism in pregnancy. In Foetus and Placenta, edited by A. Klopper and E. Diczfalusy, pp. 191-248. Blackwell, Oxford.

England, P., Lorrimer, D., Fergusson, J. C., Moffatt, A. M., and Kelly, A. M. (1974). Human placental lactogen: the watchdog of fetal distress. Lancet, 1, 5-7.

Evans, J. H., Taft, H. P., Rome, R. M., and Brown, J. B. (1972). Oestriol excretion in the pregnancy complicated by diabetes mellitus. Aust. N.Z. J. Obstet. Gynaec., 12, 95-101.

Frandsen, V. A., and Stakemann, G. (1960). Urinary excretion of estriol in pathological pregnancies. Dan. med. Bull., 7, 98-101.

Garrow, J. S., and Douglas, C. P. (1968). A rapid method for assessing intrauterine growth by radioactive selenomethionine uptake. J. Obstet. Gynaec. Brit. Cwlth, 75, 1034-1039.

Gitlin, D., and Boesman, M. (1966). Serum $a$-fetoprotein. albumin, and $\mu \mathrm{G}$-globulin in the human conceptus. J. Clin, Invest., 45, 1826-1838.

Gold, P., and Freedman, S. O. (1965). Specific carcinoembryonic antigens of the human digestive system. J. exp. Med., 122, 467-481.

Goldenberg, D. M., Tchilinguirian, N. G. O., Hansen, H. J., and Vandevoorde, J. P. (1972). Carcinoembryonic antigen present in meconium: the basis of a possible new diagnostic test of fetal distress. Amer. J. Obstet. Gynec., 113, 66-69.

Heys, R. F., Scott, J. S., Oakey, R. E., and Stitch, S. R. (1968). Urinary oestrogen in late pregnancy: oestriol excretion as a guide to impending foetal death before term. Lancet, 1 , 328-331.

Keller, P. J., Baertschi, U., Bader, P., Gerber, C., Schmid, J., Soltermann, R., and Kopper, E. (1971). Biochemical detection of fetoplacental distress in risk pregnancies. Lancet, 2, 729-731.

Klopper, A., and Gardner, J., Eds. (1973). Endocrine Factors in Labour. Mem. Soc. Endocr., 20.

Klopper, A., and Shaaban, M. M. (1974). Variability of fetoplacental steroids in maternal plasma in late pregnancy. Obstet. and Gynec., 44, 187-193.

Lauritzen, C. (1967). A clinical test for placental functional activity using DHEA-sulphate and ACTH injections in the pregnant woman. (Abstr.), Acta endocr. (Kbh.), Suppl. $119,188$.

Leek, A. E., Ruoss, C., Kitau, M. J., and Chard, T. (1975). Maternal plasma alphafetoprotein levels in the second half of normal pregnancy: relationship to fetal weight, and maternal age and parity. Brit. J. Obstet. Gynaec., 82, 669-673.

Letchworth, A. T., and Chard, T. (1972). Placental lactogen levels as a screening test for fetal distress and neonatal asphyxia. Lancet, 1, 704-706.

Lindberg, B. S. and Nilsson, B. A. (1973). Human placental lactogen (HPL) levels in abnormal pregnancies. J. Obstet. Gynaec. Brit. Cwlth, 80, 1046-1053.

Lundwall, F., and Stakemann, G. (1966). The urinary excretion of oestriol in postmaturity. Acta obstet. gynaec. scand., 45, 301-319.

Macleod, S. C., Brown, J. B., Beischer, N. A., and Smith, M. A. (1967). The value of urinary oestriol measurements during pregnancy. Aust. N.Z. J. Obstet. Gynaec., 7, 25-38.

McNeilly, A. S., Gardner, J., Bradford, D., and Chard, T. (1976). Control of placental hormone synthesis by massaction feedback. (In preparation).

Niven, P. A. R., Landon, J., and Chard, T. (1972). Placental lactogen levels as guide to outcome of threatened abortion. Brit. med.J., 3, 799-801.

Oakey, R. E. (1970). The interpretation of urinary oestrogen and pregnanediol excretion in pregnant women receiving corticosteroids. J. Obstet. Gynaec. Brit. Cwlth, 77, 922-927.

Pavlou, C., Chard, T., Landon, J., and Letchworth, A. T. (1973). Circulating levels of human placental lactogen in late pregnancy: the effect of glucose loading, smoking and exercise. Europ. J. Obstet. Gynaec. reprod. Biol., 3, 45-49.

Registrar General (1972). Statistical Review of England and Wales, 1972, Part 1, Tables, Medical, p. 465, Appendix F1, Maternal deaths .... and death rates ... 1942 to 1972 . HMSO, London.

Seppälä, M., and Ruoslahti, E. (1973). Alpha-fetoprotein: physiology and pathology during pregnancy and application to antenatal diagnosis. J. perinat. Med., 1, 104-113.

Shane, J. M., and Suzuki, K. (1974). Placental alkaline phosphatase: a review and re-evaluation of its applicability in monitoring fetoplacental function. Obstet. gynec. Surv., 29, 97-105.

Smith, K., Greene, J. W., Jr., and Touchstone, J. C. (1966). Urinary estriol determination in the management of prolonged pregnancy. Amer.J. Obstet. Gynec., 96, 901-906.

Spellacy, W. N., Teoh, E. S., Buhi, W. C., Birk, S. A., and McCreary, S. A. (1971). Value of human chorionic somatomammotropin in managing high-risk pregnancies. Amer. J. Obstet. Gynec., 109, 588-598.

Tamby Raja, R. L., Anderson, A. B. M., and Turnbull, A. C. (1974). Endocrine changes in premature labour. Brit. med.J. 4, 67-71. 
Tovey, J. E., Dawson, P. J. G., and Fellowes, K. P. (1973). Evaluation of S-benzyl-L-cystein-4'-nitroanilide as a substrate for serum cystine aminopeptidase. Clin. Chem., 19, 756-761.

Ursell, W., Brudenell, M., and Chard, T. (1973). Placental lactogen levels in diabetic pregnancy. Brit. med. J., 2, 80-82.

Ward, H., Rochman, H., Varnavides, L. A., and Whyley, G. A. (1973). Hormone and enzyme levels in normal and complicated pregnancy. Amer. J. Obstet. Gynec., 116, 1105-1113.

Ward, R. H. T., Letchworth, A. T., Niven, P. A. R., and Chard, T. (1974). Placental lactogen levels in Rhesus isoimmunization. Brit med. J., 1, 347-349.

Yousem, H., Seitchik, J., and Solomon, D. (1966). Maternal estriol excretion and fetal dysmaturity. Obstet. and Gynec., 28, 491-494. 